\title{
New fixed point theorem under $R$-contractions
}

\author{
Antonio Francisco Roldán López de Hierro ${ }^{1}$ and Naseer Shahzad ${ }^{2 *}$
}

\author{
*Correspondence: \\ nshahzad@kau.edu.sa \\ ${ }^{2}$ Operator Theory and Applications \\ Research Group, Department of \\ Mathematics, King Abdulaziz \\ University, P.O. Box 80203, Jeddah, \\ 21589, Saudi Arabia \\ Full list of author information is \\ available at the end of the article
}

\begin{abstract}
In this manuscript we introduce the notions of $R$-function and $R$-contractions, and we show an ad hoc fixed point theorem. We prove that this new kind of contractions properly includes the family of all Meir-Keeler contractions and other well-known classes of contractions that have been given very recently (for instance, those using simulation functions and manageable functions). As a consequence, our approach turns out to be appropriate to unify the treatment of different kinds of contractive nonlinear operators.
\end{abstract}

MSC: 46T99; 47H10;47H09; 54H25

Keywords: $R$-function; simulation function; manageable function; fixed point theorem

\section{Introduction}

Fixed point theory is a branch of nonlinear analysis that can be applied successfully to a wide range of contexts in social and natural sciences. Although some results had been introduced before, it is usually considered that this field of study was born in 1922, when Banach presented a celebrated theorem in order to guarantee that a nonlinear operator had a fixed point. After the appearance of the Banach contractive mapping principle, lots of generalizations, in many different frameworks, have been done. In many cases, new results that currently are being obtained involve contractivity conditions that depend on auxiliary functions (comparison functions, Geraghty functions, altering distance functions, Bianchini-Grandolfi gauge functions, etc.).

One of the extensions that have attracted much attention over the last years was due to Meir and Keeler (see [1]) who introduced in 1969 a family of contractive mappings in a new sense. Although their original notion did not depend on auxiliary functions, Lim [2] proved that a self-mapping was a Meir-Keeler contraction if, and only if, it satisfied a contractivity condition in a classical sense depending on a new class of functions (that he called L-functions). After that, several extensions of Meir-Keeler contractions have appeared (see, for instance, [3-11]).

Very recently, Khojasteh et al. (see [12]) introduced the notion of simulation function, which was later modified by Roldán-López-de-Hierro et al. in a subtle way (see [13]). The main difference with respect to previous approaches was that simulation functions depend on two variables rather than on a unique variable. And, in order to extend some results in the field of multi-valued maps, Du and Khojasteh presented the very close (but

C 2015 Roldán López de Hierro and Shahzad. This article is distributed under the terms of the Creative Commons Attribution 4.0 International License (http://creativecommons.org/licenses/by/4.0/), which permits unrestricted use, distribution, and reproduction in any medium, provided you give appropriate credit to the original author(s) and the source, provide a link to the Creative Commons license, and indicate if changes were made. 
independent) notion of manageable function (see [14]). Surprisingly, contractions that use simulation functions turned out to be Meir-Keeler contractions (see [15]).

This fact points up the difficulty in finding true extensions of Meir-Keeler contractions when we use a simple contractivity condition only involving classical terms as $d(x, y)$ and $d(T x, T y)$, where $d$ is the distance and $T: X \rightarrow X$ is the nonlinear operator.

The main aim of the present manuscript is to give a set of auxiliary functions that let us consider a true extension of Meir-Keeler contractions. To do that, we present the notion of $R$-contraction, which permits us to introduce such a large family of contractions that includes not only Meir-Keeler contractions, but Geraghty contractions, contractions depending on simulations functions and manageable functions, etc. We illustrate such kind of contractions with an example in which previous results are not applicable.

\section{Preliminaries}

In the sequel, $\mathbb{N}=\{0,1,2,3, \ldots\}$ stands for the set of all nonnegative integers. Throughout this manuscript, let $A \subseteq \mathbb{R}$ be a nonempty subset of real numbers, let $(X, d)$ be a metric space and let $T: X \rightarrow X$ be a mapping from $X$ into itself. We denote the range of $d$ by

$$
\operatorname{ran}(d)=\{d(x, y): x, y \in X\} \subseteq[0, \infty) .
$$

We say that a sequence $\left\{x_{n}\right\} \subseteq X$ is asymptotically regular on $(X, d)$ if $\left\{d\left(x_{n}, x_{n+1}\right)\right\} \rightarrow 0$.

A fixed point of $T$ is a point $x \in X$ such that $T x=x$. The iterates of the self-mapping $T$ are the mappings $\left\{T^{n}: X \rightarrow X\right\}_{n \in \mathbb{N}}$ defined by

$$
T^{0}=I_{X}, \quad T^{1}=T, \quad T^{2}=T \circ T, \quad T^{n+1}=T \circ T^{n} \quad \text { for all } n \geq 2 .
$$

Given a point $x_{0} \in X$, the Picard sequence of $T$ based on $x_{0}$ is the sequence $\left\{x_{n}\right\}_{n \in \mathbb{N}}$ given by $x_{n+1}=T x_{n}$ for all $n \in \mathbb{N}$. Notice that $x_{n}=T^{n} x_{0}$ for all $n \in \mathbb{N}$.

Following $[16,17]$, we say that $T$ is a weakly Picard operator if, for all $x_{0} \in X$, the Picard sequence of $T$ based on $x_{0}$ converges to a fixed point of $T$. Furthermore, $T$ is a Picard operator if it is a weakly Picard operator and it has a unique fixed point. In such a case, if $z_{0}$ is the unique fixed point of $T$, then $\left\{T^{n} x_{0}\right\} \rightarrow z_{0}$ for all $x_{0} \in X$.

A function $\phi:[0, \infty) \longrightarrow[0, \infty)$ is lower semi-continuous if

$$
\phi(L) \leq \liminf _{t \rightarrow L} \phi(t) \quad \text { for all } L \in[0, \infty)
$$

A Geraghty function is a function $\phi:[0, \infty) \rightarrow[0,1)$ such that if $\left\{t_{n}\right\} \subset[0, \infty)$ and $\left\{\phi\left(t_{n}\right)\right\} \rightarrow 1$, then $\left\{t_{n}\right\} \rightarrow 0$. A Geraghty contraction (see [18]) is a mapping $T: X \rightarrow X$ such that

$$
d(T x, T y) \leq \phi(d(x, y)) d(x, y) \text { for all } x, y \in X,
$$

where $\phi$ is a Geraghty function.

\subsection{Simulation functions and manageable functions}

The notion of simulation function was introduced by Khojasteh et al. in [12] as follows.

Definition 1 (Khojasteh et al. [12]) A simulation function is a mapping $\zeta:[0, \infty) \times$ $[0, \infty) \rightarrow \mathbb{R}$ satisfying the following conditions: 
$\left(\zeta_{1}\right) \zeta(0,0)=0$;

$\left(\zeta_{2}\right) \zeta(t, s)<s-t$ for all $t, s>0$;

$\left(\zeta_{3}\right)$ if $\left\{t_{n}\right\},\left\{s_{n}\right\}$ are sequences in $(0, \infty)$ such that $\lim _{n \rightarrow \infty} t_{n}=\lim _{n \rightarrow \infty} s_{n}>0$, then

$$
\limsup _{n \rightarrow \infty} \zeta\left(t_{n}, s_{n}\right)<0
$$

The third condition is symmetric in both arguments of $\zeta$ but, in proofs, this property is not necessary. In fact, in practise, the arguments of $\zeta$ have different meanings and they play different roles. Then, Roldán-López-de-Hierro et al. slightly modified the previous definition in order to highlight this difference and to enlarge the family of all simulation functions.

Definition 2 (Roldán-López-de-Hierro et al. [13]) A simulation function is a mapping $\zeta:[0, \infty) \times[0, \infty) \rightarrow \mathbb{R}$ satisfying the following conditions:

$\left(\zeta_{1}\right) \zeta(0,0)=0$;

$\left(\zeta_{2}\right) \zeta(t, s)<s-t$ for all $t, s>0$;

$\left(\zeta_{3}\right)$ if $\left\{t_{n}\right\},\left\{s_{n}\right\}$ are sequences in $(0, \infty)$ such that $\lim _{n \rightarrow \infty} t_{n}=\lim _{n \rightarrow \infty} s_{n}>0$ and $t_{n}<s_{n}$ for all $n \in \mathbb{N}$, then

$$
\limsup _{n \rightarrow \infty} \zeta\left(t_{n}, s_{n}\right)<0
$$

Let $\mathcal{Z}$ be the family of all simulation functions $\zeta:[0, \infty) \times[0, \infty) \rightarrow \mathbb{R}$.

Every simulation function in the original Khojasteh et al.'s sense (Definition 1) is also a simulation function in our sense (Definition 2), but the converse is not true (see [13]).

Definition 3 (Khojasteh et al. [12], Roldán-López-de-Hierro et al. [13]) Let $(X, d)$ be a metric space and let $T: X \rightarrow X$ be a self-mapping. We say that $T$ is a $\mathcal{Z}$-contraction if there exists $\zeta \in \mathcal{Z}$ such that

$$
\zeta(d(T x, T y), d(x, y)) \geq 0 \quad \text { for all } x, y \in X \text { such that } x \neq y .
$$

In 2014, Du and Khojasteh [14] introduced the concept of manageable functions. They showed that many known results can be deduced of some local constraints related to manageable functions.

Definition 4 (Du and Khojasteh [14]) A function $\eta: \mathbb{R} \times \mathbb{R} \rightarrow \mathbb{R}$ is called manageable if the following conditions hold:

$\left(\eta_{1}\right) \eta(t, s)<s-t$ for all $s, t>0$.

$\left(\eta_{2}\right)$ For any bounded sequence $\left\{t_{n}\right\} \subset(0, \infty)$ and any nonincreasing sequence $\left\{s_{n}\right\} \subset$ $(0, \infty)$, it holds

$$
\limsup _{n \rightarrow \infty} \frac{t_{n}+\eta\left(t_{n}, s_{n}\right)}{s_{n}}<1
$$

We denote the set of all manageable functions by $\widehat{\operatorname{Man}(\mathrm{R})}$. 
Several examples of simulation functions and manageable functions can be found on [12-14].

Example 5 If $k \in(0,1)$, then the function $\eta: \mathbb{R} \times \mathbb{R} \rightarrow \mathbb{R}$ given by $\eta(t, s)=k s-t$ for all $t, s \in \mathbb{R}$, is a manageable function, and its restriction to $[0, \infty) \times[0, \infty)$ is a simulation function.

The notion of manageable function was introduced in order to study multi-valued contractions. Next, we particularize such a notion to single-valued mappings.

Definition 6 (Khojasteh et al. [12]) Let $(X, d)$ be a metric space and let $T: X \rightarrow X$ be a self-mapping. We say that $T$ is a $\widehat{\operatorname{Man}(\mathrm{R})}$-contraction if there exists $\eta \in \widehat{\operatorname{Man}(\mathrm{R})}$ such that $\eta\left(d\left(T x, T^{2} x\right), d(x, T x)\right) \geq 0$ for all $x \in X$.

\subsection{Meir-Keeler contractions}

Meir and Keeler generalized the Banach theorem in the following way.

Definition 7 (Meir and Keeler [1]) A Meir-Keeler contraction is a mapping $T: X \rightarrow X$ from a metric space $(X, d)$ into itself such that for all $\varepsilon>0$, there exists $\delta>0$ verifying that if $x, y \in X$ and $\varepsilon \leq d(x, y)<\varepsilon+\delta$, then $d(T x, T y)<\varepsilon$.

Meir-Keeler contractions have attracted much attention in the last years (see, for instance, [3-11]). Lim characterized this kind of mappings in terms of a contractivity condition using the following class of auxiliary functions.

Definition 8 (Lim [2]) A function $\phi:[0, \infty) \rightarrow[0, \infty)$ will be called an L-function if

(a) $\phi(0)=0$,

(b) $\phi(t)>0$ for all $t>0$, and

(c) for all $\varepsilon>0$, there exists $\delta>0$ such that $\phi(t) \leq \varepsilon$ for all $t \in[\varepsilon, \varepsilon+\delta]$.

Theorem 9 (Lim [2], Theorem 1) Let $(X, d)$ be a metric space and let $T: X \rightarrow X$ be a selfmapping. Then $T$ is a Meir-Keeler mapping if, and only if, there exists an (nondecreasing, right-continuous) L-map $\phi$ such that

$$
d(T x, T y)<\phi(d(x, y)) \quad \text { for all } x, y \in X \text { verifying } d(x, y)>0 .
$$

Using a result of Chu and Diaz [19], Meir and Keeler [1] proved that every Meir-Keeler contraction from a complete metric space into itself has a unique fixed point.

For our purposes, we highlight the following properties of $L$-functions and Meir-Keeler contractions.

Lemma 10 Every L-function $\phi$ satisfies the following properties:

(1) $\phi(t) \leq t$ for all $t \in[0, \infty)$.

(2) For all $\varepsilon>0$, there exists $\delta>0$ such that $\phi(t) \leq \varepsilon$ for all $t \in[0, \varepsilon+\delta]$.

Proof (1) If $t=0$, then $\phi(0)=0$. And if $t>0$, using $\varepsilon=t>0$, we deduce, from (c), that $\phi(t)=\phi(\varepsilon) \leq \varepsilon=t$. 
(2) Let $\varepsilon>0$ be arbitrary and let $\delta>0$ be given by (c). Then, for all $t \in[0, \varepsilon)$, we have that $\phi(t) \leq t<\varepsilon$.

The following result is useful to guarantee that a self-mapping is not a Meir-Keeler contraction.

Proposition 11 Let $T: X \rightarrow X$ be a self-mapping from a metric space $(X, d)$ into itself and assume that there exist $L>0$ and two sequences $\left\{x_{n}\right\},\left\{y_{n}\right\} \subseteq X$ such that

$$
\begin{aligned}
& d\left(T x_{n}, T y_{n}\right)=L<d\left(x_{n}, y_{n}\right) \text { for all } n \in \mathbb{N} \text { and } \\
& \left\{d\left(x_{n}, y_{n}\right)\right\} \rightarrow L .
\end{aligned}
$$

Then $T$ is not a Meir-Keeler contraction.

Proof Reasoning by contradiction, assume that $T$ is a Meir-Keeler contraction. By Theorem 9 , there exists an $L$-map $\phi:[0, \infty) \times[0, \infty) \rightarrow[0, \infty)$ such that

$$
d(T x, T y)<\phi(d(x, y)) \quad \text { for all } x, y \in X \text { verifying } d(x, y)>0
$$

As a consequence,

$$
\phi(L) \leq L=d\left(T x_{n}, T y_{n}\right)<\phi\left(d\left(x_{n}, y_{n}\right)\right) \leq d\left(x_{n}, y_{n}\right) \quad \text { for all } n \in \mathbb{N}
$$

Using $\varepsilon=L>0$, taking into account that $T$ is a Meir-Keeler contraction, there exists $\delta>0$ such that

$$
\phi(t)<L \quad \text { for all } t \in[L, L+\delta] .
$$

As $\left\{d\left(x_{n}, y_{n}\right)\right\} \rightarrow L$ and $d\left(x_{n}, y_{n}\right)>L$ for all $n \in \mathbb{N}$, there exists $n_{0} \in \mathbb{N}$ such that $L<$ $d\left(x_{n_{0}}, y_{n_{0}}\right)<L+\delta$. Then $\phi\left(d\left(x_{n_{0}}, y_{n_{0}}\right)\right)<L$, which contradicts the fact that $L<\phi\left(d\left(x_{n_{0}}, y_{n_{0}}\right)\right)$. As a result, $T$ cannot be a Meir-Keeler contraction.

\section{R-Functions and $R$-contractions}

In this section we introduce the family of auxiliary functions we will use to present a new kind of contractive mappings. We will also show that this family contains several classes of contractive mappings, including Meir-Keeler contractions.

\subsection{The family of $R$-functions}

Definition 12 Let $A \subseteq \mathbb{R}$ be a nonempty subset and let $\varrho: A \times A \rightarrow \mathbb{R}$ be a function. We say that $\varrho$ is an $R$-function if it satisfies the following two conditions.

$\left(\varrho_{1}\right)$ If $\left\{a_{n}\right\} \subset(0, \infty) \cap A$ is a sequence such that $\varrho\left(a_{n+1}, a_{n}\right)>0$ for all $n \in \mathbb{N}$, then $\left\{a_{n}\right\} \rightarrow 0$.

$\left(\varrho_{2}\right)$ If $\left\{a_{n}\right\},\left\{b_{n}\right\} \subset(0, \infty) \cap A$ are two sequences converging to the same limit $L \geq 0$ and verifying that $L<a_{n}$ and $\varrho\left(a_{n}, b_{n}\right)>0$ for all $n \in \mathbb{N}$, then $L=0$.

We denote by $R_{A}$ the family of all $R$-functions whose domain is $A \times A$.

In some cases, given a function $\varrho: A \times A \rightarrow \mathbb{R}$, we will also consider the following property. 
$\left(\varrho_{3}\right)$ If $\left\{a_{n}\right\},\left\{b_{n}\right\} \subset(0, \infty) \cap A$ are two sequences such that $\left\{b_{n}\right\} \rightarrow 0$ and $\varrho\left(a_{n}, b_{n}\right)>0$ for all $n \in \mathbb{N}$, then $\left\{a_{n}\right\} \rightarrow 0$.

Remark 13 Notice that conditions $\left(\varrho_{1}\right),\left(\varrho_{2}\right)$ and $\left(\varrho_{3}\right)$ establish that if there exist sequences verifying some assumptions, then a thesis must hold. However, we point out that if such kind of sequences does not exist, then conditions $\left(\varrho_{1}\right),\left(\varrho_{2}\right)$ and $\left(\varrho_{3}\right)$ hold.

Proposition 14 If $\varrho(t, s) \leq s-t$ for all $t, s \in A \cap(0, \infty)$, then $\left(\varrho_{3}\right)$ holds.

Proof Assume that $\left\{a_{n}\right\},\left\{b_{n}\right\} \subset(0, \infty) \cap A$ are two sequences such that $\left\{b_{n}\right\} \rightarrow 0$ and $\varrho\left(a_{n}, b_{n}\right)>0$ for all $n \in \mathbb{N}$. Since $a_{n}, b_{n} \in(0, \infty) \cap A$, then $0<\varrho\left(a_{n}, b_{n}\right) \leq b_{n}-a_{n}$ for all $n \in \mathbb{N}$. As a consequence, $0<a_{n}<b_{n}$ for all $n \in \mathbb{N}$, which means that $\left\{a_{n}\right\} \rightarrow 0$.

Firstly, we show some examples.

Lemma 15 Every simulation function is an R-function that also verifies $\left(\varrho_{3}\right)$.

Proof Let $\zeta:[0, \infty) \times[0, \infty) \rightarrow \mathbb{R}$ be a simulation function.

$\left(\varrho_{1}\right)$ Let $\left\{a_{n}\right\} \subset(0, \infty)$ be a sequence such that $\zeta\left(a_{n+1}, a_{n}\right)>0$ for all $n \in \mathbb{N}$. Therefore, $0<$ $\zeta\left(a_{n+1}, a_{n}\right)<a_{n}-a_{n+1}$ for all $n \in \mathbb{N}$. Hence, $\left\{a_{n}\right\}$ is a strictly decreasing sequence of positive real numbers, so it is convergent. Let $L \geq 0$ be its limit. To prove that $L=0$, assume, by contradiction, that $L>0$. Let $t_{n}=a_{n+1}$ and $s_{n}=a_{n}$ for all $n \in \mathbb{N}$. Then $\left\{t_{n}\right\}$ and $\left\{s_{n}\right\}$ are convergent to $L$ and $t_{n}<s_{n}$ for all $n \in \mathbb{N}$. By condition $\left(\zeta_{3}\right)$,

$$
0 \leq \limsup _{n \rightarrow \infty} \zeta\left(a_{n+1}, a_{n}\right)=\limsup _{n \rightarrow \infty} \zeta\left(t_{n}, s_{n}\right)<0,
$$

which is a contradiction. Then $\left\{a_{n}\right\} \rightarrow L=0$.

$\left(\varrho_{2}\right)$ Let $\left\{a_{n}\right\},\left\{b_{n}\right\} \subset(0, \infty)$ be two sequences converging to the same limit $L \geq 0$ and verifying that $L<a_{n}$ and $\zeta\left(a_{n}, b_{n}\right)>0$ for all $n \in \mathbb{N}$. To prove that $L=0$, assume, by contradiction, that $L>0$. On the one hand, by $\left(\zeta_{2}\right), 0<\zeta\left(a_{n}, b_{n}\right)=b_{n}-a_{n}$, so $L<a_{n}<b_{n}$ for all $n \in \mathbb{N}$. On the other hand, by condition $\left(\zeta_{3}\right)$, we have that

$$
0 \leq \limsup _{n \rightarrow \infty} \zeta\left(a_{n}, b_{n}\right)<0,
$$

which is a contradiction. Then $L=0$.

$\left(\varrho_{3}\right)$ Let $\left\{a_{n}\right\},\left\{b_{n}\right\} \subset(0, \infty)$ be two sequences such that $\left\{b_{n}\right\} \rightarrow 0$ and $\zeta\left(a_{n}, b_{n}\right) \geq 0$ for all $n \in \mathbb{N}$. Since $\zeta$ is a simulation function, $0 \leq \zeta\left(a_{n}, b_{n}\right)<b_{n}-a_{n}$ for all $n \in \mathbb{N}$. Hence, $0<a_{n}<b_{n}$ for all $n \in \mathbb{N}$, which means that $\left\{a_{n}\right\} \rightarrow 0$.

Lemma 16 Every manageable function is an R-function that also verifies $\left(\varrho_{3}\right)$.

Proof Let $\eta: \mathbb{R} \times \mathbb{R} \rightarrow \mathbb{R}$ be a manageable function.

$\left(\varrho_{1}\right)$ Let $\left\{a_{n}\right\} \subset(0, \infty)$ be a sequence such that $\eta\left(a_{n+1}, a_{n}\right)>0$ for all $n \in \mathbb{N}$. Therefore, $0<$ $\eta\left(a_{n+1}, a_{n}\right)<a_{n}-a_{n+1}$ for all $n \in \mathbb{N}$. Hence, $\left\{a_{n}\right\}$ is a strictly decreasing sequence of positive real numbers, so it is convergent. Let $L \geq 0$ be its limit. To prove that $L=0$, assume, by contradiction, that $L>0$. Let $t_{n}=a_{n+1}$ and $s_{n}=a_{n}$ for all $n \in \mathbb{N}$. Then $\left\{t_{n}\right\}$ is a bounded 
sequence (in fact, it is convergent) and $\left\{s_{n}\right\}$ is a nonincreasing sequence (convergent to $L$ ). By condition $\left(\eta_{2}\right)$,

$$
\limsup _{n \rightarrow \infty} \frac{a_{n+1}+\eta\left(a_{n+1}, a_{n}\right)}{a_{n}}=\limsup _{n \rightarrow \infty} \frac{t_{n}+\eta\left(t_{n}, s_{n}\right)}{s_{n}}<1 .
$$

Taking into account that

$$
\frac{a_{n+1}}{a_{n}} \leq \frac{a_{n+1}+\eta\left(a_{n+1}, a_{n}\right)}{a_{n}} \text { for all } n \in \mathbb{N}
$$

we deduce that

$$
1=\frac{L}{L}=\lim _{n \rightarrow \infty} \frac{a_{n+1}}{a_{n}}=\limsup _{n \rightarrow \infty} \frac{a_{n+1}}{a_{n}} \leq \limsup _{n \rightarrow \infty} \frac{a_{n+1}+\eta\left(a_{n+1}, a_{n}\right)}{a_{n}}<1,
$$

which is a contradiction. Then $\left\{a_{n}\right\} \rightarrow L=0$.

$\left(\varrho_{2}\right)$ Let $\left\{a_{n}\right\},\left\{b_{n}\right\} \subset(0, \infty)$ be two sequences converging to the same limit $L \geq 0$ and verifying that $L<a_{n}$ and $\zeta\left(a_{n}, b_{n}\right)>0$ for all $n \in \mathbb{N}$. To prove that $L=0$, assume, by contradiction, that $L>0$. On the one hand, by $\left(\eta_{1}\right), 0<\eta\left(a_{n}, b_{n}\right)=b_{n}-a_{n}$, so $L<a_{n}<b_{n}$ for all $n \in \mathbb{N}$. As $\left\{b_{n}\right\} \rightarrow L$ and $L<b_{n}$ for all $n \in \mathbb{N}$, then there exists a partial subsequence $\left\{b_{n(k)}\right\}_{k \in \mathbb{N}}$ of $\left\{b_{n}\right\}$ such that $\left\{b_{n(k)}\right\}$ is strictly decreasing, that is,

$$
L<b_{n(k+1)}<b_{n(k)} \quad \text { for all } k \in \mathbb{N} .
$$

Hence $\left\{a_{n(k)}\right\}$ is a bounded sequence (in fact, it is convergent to $L$ ) and $\left\{b_{n(k)}\right\}$ is a nonincreasing sequence. By condition $\left(\eta_{2}\right)$, we have that

$$
\limsup _{n \rightarrow \infty} \frac{b_{n(k)}+\eta\left(a_{n(k)}, b_{n(k)}\right)}{a_{n(k)}}<1 .
$$

Taking into account that

$$
\frac{b_{n(k)}}{a_{n(k)}} \leq \frac{b_{n(k)}+\eta\left(a_{n(k)}, b_{n(k)}\right)}{a_{n(k)}} \quad \text { for all } n \in \mathbb{N}
$$

we deduce that

$$
1=\frac{L}{L}=\lim _{n \rightarrow \infty} \frac{b_{n(k)}}{a_{n(k)}}=\limsup _{n \rightarrow \infty} \frac{b_{n(k)}}{a_{n(k)}} \leq \limsup _{n \rightarrow \infty} \frac{b_{n(k)}+\eta\left(a_{n(k)}, b_{n(k)}\right)}{a_{n(k)}}<1,
$$

which is a contradiction. Then $L=0$.

$\left(\varrho_{3}\right)$ Let $\left\{a_{n}\right\},\left\{b_{n}\right\} \subset(0, \infty)$ be two sequences such that $\left\{b_{n}\right\} \rightarrow 0$ and $\eta\left(a_{n}, b_{n}\right) \geq 0$ for all $n \in \mathbb{N}$. Since $\eta$ is a manageable function, $0 \leq \eta\left(a_{n}, b_{n}\right)<b_{n}-a_{n}$ for all $n \in \mathbb{N}$. Hence, $0<a_{n}<b_{n}$ for all $n \in \mathbb{N}$, which means that $\left\{a_{n}\right\} \rightarrow 0$.

Lemmas 15 and 16 provide us with a wide range of $R$-functions taking into account examples given in $[12,14]$. In the following examples we show that the notion of $R$-function is more general than the previous ones.

Example 17 Given $\lambda \in(0,1)$, let $\varrho:[0,1] \times[0,1] \rightarrow \mathbb{R}$ be the function given by $\varrho(t, s)=$ $\lambda s-t$ for all $t, s \in[0,1]$. Then $\varrho$ is an $R$-function, but it is not a simulation function neither a manageable function because its domain is neither $[0, \infty) \times[0, \infty)$ nor $\mathbb{R} \times \mathbb{R}$. 
Example 18 Let $\varrho:[0, \infty) \times[0, \infty) \rightarrow \mathbb{R}$ be the function defined, for all $t, s \in[0, \infty)$, by

$$
\varrho(t, s)= \begin{cases}\frac{1}{2} s-t, & \text { if } t<s \\ 0, & \text { if } t \geq s\end{cases}
$$

Let us show that $\varrho$ is an $R$-function on $[0, \infty)$ which also satisfies condition $\left(\varrho_{3}\right)$.

$\left(\varrho_{1}\right)$ Assume that $\left\{a_{n}\right\} \subset(0, \infty)$ is a sequence such that $\varrho\left(a_{n+1}, a_{n}\right)>0$ for all $n \in \mathbb{N}$. Therefore $a_{n+1}<a_{n}$ and

$$
0<\varrho\left(a_{n+1}, a_{n}\right)=\frac{a_{n}}{2}-a_{n+1} \quad \text { for all } n \in \mathbb{N}
$$

As a consequence, $0<a_{n+1}<a_{n} / 2$ for all $n \in \mathbb{N}$, which implies that $\left\{a_{n}\right\} \rightarrow 0$.

$\left(\varrho_{2}\right)$ Assume that $\left\{a_{n}\right\},\left\{b_{n}\right\} \subset(0, \infty)$ are two sequences converging to the same limit $L \geq 0$ and verifying that $L<a_{n}$ and $\varrho\left(a_{n}, b_{n}\right)>0$ for all $n \in \mathbb{N}$. Hence

$$
a_{n}<b_{n} \quad \text { and } \quad 0<\varrho\left(a_{n}, b_{n}\right)=\frac{b_{n}}{2}-a_{n} \quad \text { for all } n \in \mathbb{N} \text {. }
$$

As a consequence, $2 a_{n}<b_{n}$ for all $n \in \mathbb{N}$. Letting $n \rightarrow \infty$, we deduce that $0 \leq 2 L \leq L$, which means that $L=0$.

$\left(\varrho_{3}\right)$ Let $\left\{a_{n}\right\},\left\{b_{n}\right\} \subset(0, \infty) \cap A$ be two sequences such that $\left\{b_{n}\right\} \rightarrow 0$ and $\varrho\left(a_{n}, b_{n}\right)>0$ for all $n \in \mathbb{N}$. Therefore, $0<a_{n}<b_{n}$, which implies that $\left\{a_{n}\right\} \rightarrow 0$.

As a consequence, $\varrho$ is an $R$-function on $[0, \infty)$ which also satisfies condition $\left(\varrho_{3}\right)$. However, $\varrho$ is not a simulation function because if we take $t_{n}=s_{n}=1$ for all $n \in \mathbb{N}$, then $\left\{t_{n}\right\} \rightarrow 1$, $\left\{s_{n}\right\} \rightarrow 1$ but $\varrho\left(t_{n}, s_{n}\right)=0$ for all $n \in \mathbb{N}$, which implies that $\varrho$ does not verify condition $\left(\zeta_{3}\right)$. The same argument guarantees that $\varrho$, defined from $\mathbb{R} \times \mathbb{R}$ into $\mathbb{R}$, is not a manageable function.

Proposition 19 If $\varrho \in R_{A}$, then $\varrho(a, a) \leq 0$ for all $a \in(0, \infty) \cap A$.

Proof By contradiction, assume that there exists $a \in(0, \infty) \cap A$ such that $\varrho(a, a)>0$. Let us define $a_{n}=a$ for all $n \in \mathbb{N}$. Therefore $\varrho\left(a_{n+1}, a_{n}\right)=\varrho(a, a)>0$ for all $n \in \mathbb{N}$. Condition $\left(\varrho_{1}\right)$ implies that $\left\{a_{n}\right\} \rightarrow 0$, which contradicts the fact that $a>0$.

Functions taking values greater than or equal to an $R$-function can be an $R$-function.

Proposition 20 If $\varrho \in R_{A}$ and $\lambda>0$, then $\varrho_{\lambda}: A \times A \rightarrow \mathbb{R}$, defined by $\varrho_{\lambda}(t, s)=\lambda \varrho(t, s)$ for all $t, s \in A$, is also an $R$-function. And if $\varrho$ satisfies $\left(\varrho_{3}\right)$, then $\varrho_{\lambda}$ also satisfies it.

An interesting subclass of the family of $R$-functions can be considered involving $L$ functions as follows.

Theorem 21 Given an L-function $\phi:[0, \infty) \rightarrow[0, \infty)$, let $\varrho_{\phi}:[0, \infty) \times[0, \infty) \rightarrow \mathbb{R}$ be the function defined by

$$
\varrho_{\phi}(t, s)=\phi(s)-t \quad \text { for all } t, s \in[0, \infty)
$$

Then $\varrho_{\phi}$ is an $R$-function on $[0, \infty)$. Furthermore, $\varrho_{\phi}$ satisfies condition $\left(\varrho_{3}\right)$. 
Proof $\left(\varrho_{1}\right)$ Assume that $\left\{a_{n}\right\} \subset(0, \infty)$ is a sequence such that $\varrho_{\phi}\left(a_{n+1}, a_{n}\right)>0$ for all $n \in \mathbb{N}$. Therefore,

$$
0<\varrho_{\phi}\left(a_{n+1}, a_{n}\right)=\phi\left(a_{n}\right)-a_{n+1},
$$

which means that $a_{n+1}<\phi\left(a_{n}\right)$ for all $n \in \mathbb{N}$. Using item (1) of Lemma 10,

$$
a_{n+1}<\phi\left(a_{n}\right) \leq a_{n} \quad \text { for all } n \in \mathbb{N} \text {. }
$$

Hence, $\left\{a_{n}\right\}$ is a strictly decreasing sequence of positive real numbers. Let $L \geq 0$ be its limit. In order to prove that $L=0$, assume that $L>0$. Therefore,

$$
L<a_{n+1}<\phi\left(a_{n}\right) \leq a_{n} \text { for all } n \in \mathbb{N} \text {. }
$$

As $\phi$ is an $L$-function, using $\varepsilon=L>0$, there exists $\delta>0$ such that

$$
\phi(t) \leq \varepsilon=L \quad \text { for all } t \in[\varepsilon, \varepsilon+\delta]=[L, L+\delta] .
$$

Since $\left\{a_{n}\right\} \searrow L^{+}$, there exists $n_{0} \in \mathbb{N}$ such that $a_{n_{0}}<L+\delta$. Since $L<a_{n_{0}}<L+\delta$, then (2) implies that $\phi\left(a_{n_{0}}\right) \leq L$, which yields the contradiction

$$
\phi\left(a_{n_{0}}\right) \leq L<a_{n_{0}+1}<\phi\left(a_{n_{0}}\right) .
$$

As a consequence, $L=0$.

$\left(\varrho_{2}\right)$ Assume that $\left\{a_{n}\right\},\left\{b_{n}\right\} \subset(0, \infty)$ are two sequences converging to the same limit $L \geq 0$ and verifying that $L<a_{n}$ and $\varrho_{\phi}\left(a_{n}, b_{n}\right)>0$ for all $n \in \mathbb{N}$. In order to prove that $L=0$, assume that $L>0$. Therefore,

$$
0<\varrho_{\phi}\left(a_{n}, b_{n}\right)=\phi\left(b_{n}\right)-a_{n} .
$$

As a consequence,

$$
L<a_{n}<\phi\left(b_{n}\right) \leq b_{n} \quad \text { for all } n \in \mathbb{N} .
$$

As $\phi$ is an $L$-function, using $\varepsilon=L>0$, there exists $\delta>0$ such that

$$
\phi(t) \leq \varepsilon=L \quad \text { for all } t \in[\varepsilon, \varepsilon+\delta]=[L, L+\delta] .
$$

Since $\left\{b_{n}\right\} \backslash L^{+}$, there exists $n_{0} \in \mathbb{N}$ such that $L<b_{n_{0}}<L+\delta$. And, by using (3), we deduce that $\phi\left(b_{n_{0}}\right) \leq L$, which is a contradiction because $\phi\left(b_{n_{0}}\right) \leq L<a_{n_{0}}<\phi\left(b_{n_{0}}\right)$. Thus, $L=0$.

$\left(\varrho_{3}\right)$ Let $\left\{a_{n}\right\},\left\{b_{n}\right\} \subset(0, \infty) \cap A$ be two sequences such that $\left\{b_{n}\right\} \rightarrow 0$ and $\varrho_{\phi}\left(a_{n}, b_{n}\right)>0$ for all $n \in \mathbb{N}$. Therefore, by item (1) of Lemma 10, $0<\varrho_{\phi}\left(a_{n}, b_{n}\right)=\phi\left(b_{n}\right)-a_{n} \leq b_{n}-a_{n}$, so $0<a_{n}<b_{n}$ for all $n \in \mathbb{N}$, which implies that $\left\{a_{n}\right\} \rightarrow 0$.

Theorem 22 Let $\psi, \varphi:[0, \infty) \rightarrow[0, \infty)$ be two functions such that $\psi$ is nondecreasing and continuous from the right, $\varphi$ is lower semi-continuous and $\varphi^{-1}(\{0\})=\{0\}$. Let $\varrho_{\psi, \varphi}$ : 
$[0, \infty) \times[0, \infty) \rightarrow \mathbb{R}$ be the function defined by

$$
\varrho_{\psi, \varphi}(t, s)=\psi(s)-\varphi(s)-\psi(t) \quad \text { for all } t, s \in[0, \infty)
$$

Then $\varrho_{\psi, \varphi}$ is an $R$-function on $[0, \infty)$. Furthermore, $\varrho_{\psi, \varphi}$ satisfies condition $\left(\varrho_{3}\right)$.

Proof First of all, we show that, for $t, s \in[0, \infty)$,

$$
s>0, \quad \varrho_{\psi, \varphi}(t, s) \geq 0 \quad \Rightarrow \quad t<s
$$

Indeed, assume that $\varrho_{\psi, \varphi}(t, s) \geq 0$ and $t \geq s$, and we are going to show that $s=0$. As $\psi$ is nondecreasing,

$$
\psi(s) \leq \psi(t) \leq \psi(s)-\varphi(s) \leq \psi(s)
$$

which implies that $\varphi(s)=0$. Hence $s=0$, which contradicts the fact that $s>0$. Thus, $t<s$.

$\left(\varrho_{1}\right)$ Assume that $\left\{a_{n}\right\} \subset(0, \infty)$ is a sequence such that $\varrho_{\psi, \varphi}\left(a_{n+1}, a_{n}\right)>0$ for all $n \in \mathbb{N}$. By (4), $a_{n+1}<a_{n}$ for all $n \in \mathbb{N}$. Hence, $\left\{a_{n}\right\}$ is a strictly decreasing sequence of positive real numbers. Let $L \geq 0$ be its limit. Then $L<a_{n}$ for all $n \in \mathbb{N}$. In order to prove that $L=0$, assume that $L>0$. Therefore, for $n \in \mathbb{N}$,

$$
\begin{aligned}
0 & <\varrho_{\psi, \varphi}\left(a_{n+1}, a_{n}\right)=\psi\left(a_{n}\right)-\varphi\left(a_{n}\right)-\psi\left(a_{n+1}\right) \\
& \Rightarrow \quad 0 \leq \varphi\left(a_{n}\right)<\psi\left(a_{n}\right)-\psi\left(a_{n+1}\right) .
\end{aligned}
$$

As $\psi$ is continuous from the right and $\left\{a_{n}\right\} \searrow L^{+}$, letting $n \rightarrow \infty$ we deduce that $\lim _{n \rightarrow \infty} \varphi\left(a_{n}\right)=0$. And as $\varphi$ is lower semi-continuous,

$$
0 \leq \varphi(L) \leq \liminf _{r \rightarrow L} \varphi(r)=\lim _{n \rightarrow \infty} \varphi\left(a_{n}\right)=0 .
$$

Hence $\varphi(L)=0$, so $L=0$.

$\left(\varrho_{2}\right)$ Assume that $\left\{a_{n}\right\},\left\{b_{n}\right\} \subset(0, \infty)$ are two sequences converging to the same limit $L \geq 0$ and verifying that $L<a_{n}$ and $\varrho_{\psi, \varphi}\left(a_{n}, b_{n}\right)>0$ for all $n \in \mathbb{N}$. By $(4), L<a_{n}<b_{n}$ for all $n \in \mathbb{N}$. In order to prove that $L=0$, assume that $L>0$. Therefore,

$$
0<\varrho_{\psi, \varphi}\left(a_{n}, b_{n}\right)=\psi\left(b_{n}\right)-\varphi\left(b_{n}\right)-\psi\left(a_{n}\right) \quad \Rightarrow \quad 0 \leq \varphi\left(b_{n}\right)<\psi\left(b_{n}\right)-\psi\left(a_{n}\right) .
$$

As $\psi$ is continuous from the right and $\left\{b_{n}\right\} \searrow L^{+}$, letting $n \rightarrow \infty$ we deduce that $\lim _{n \rightarrow \infty} \varphi\left(b_{n}\right)=0$. And as $\varphi$ is lower semi-continuous,

$$
0 \leq \varphi(L) \leq \liminf _{r \rightarrow L} \varphi(r)=\lim _{n \rightarrow \infty} \varphi\left(b_{n}\right)=0 .
$$

Hence $\varphi(L)=0$, so $L=0$. 
$\left(\varrho_{3}\right)$ Let $\left\{a_{n}\right\},\left\{b_{n}\right\} \subset(0, \infty) \cap A$ be two sequences such that $\left\{b_{n}\right\} \rightarrow 0$ and $\varrho_{\phi}\left(a_{n}, b_{n}\right)>0$ for all $n \in \mathbb{N}$. By condition (4), $0<a_{n}<b_{n}$ for all $n \in \mathbb{N}$, which implies that $\left\{a_{n}\right\} \rightarrow 0$.

Another example, involving Geraghty functions, is the next statement.

Lemma 23 If $\phi:[0, \infty) \rightarrow[0,1)$ is a Geraghty function, then $\varrho_{\phi}^{\prime}:[0, \infty) \times[0, \infty) \rightarrow \mathbb{R}$, defined by

$$
\varrho_{\phi}^{\prime}(t, s)=\phi(s) s-t \quad \text { for all } t, s \in[0, \infty) \text {, }
$$

is an $R$-function on $[0, \infty)$ satisfying condition $\left(\varrho_{3}\right)$.

Proof $\left(\varrho_{1}\right)$ Assume that $\left\{a_{n}\right\} \subset(0, \infty)$ is a sequence such that $\varrho_{\phi}^{\prime}\left(a_{n+1}, a_{n}\right)>0$ for all $n \in \mathbb{N}$. Therefore,

$$
0<\varrho_{\phi}^{\prime}\left(a_{n+1}, a_{n}\right)=\phi\left(a_{n}\right) a_{n}-a_{n+1} .
$$

As $a_{n}>0$ and $\phi\left(a_{n}\right)<1$ for all $n \in \mathbb{N}$, then

$$
a_{n+1}<\phi\left(a_{n}\right) a_{n}<a_{n} \quad \text { for all } n \in \mathbb{N} \text {. }
$$

Hence, $\left\{a_{n}\right\}$ is a strictly decreasing sequence of positive real numbers. Let $L \geq 0$ be its limit. In order to prove that $L=0$, assume that $L>0$. Therefore,

$$
0<L<a_{n+1}<\phi\left(a_{n}\right) a_{n}<a_{n} \text { for all } n \in \mathbb{N} \text {. }
$$

Letting $n \rightarrow \infty$ in the last inequalities, we deduce that $\lim _{n \rightarrow \infty} \phi\left(a_{n}\right)=1$. As $\phi$ is a Geraghty function, it follows that $L=\lim _{n \rightarrow \infty} a_{n}=0$, which contradicts $L>0$. Then necessarily $L=0$.

$\left(\varrho_{2}\right)$ Assume that $\left\{a_{n}\right\},\left\{b_{n}\right\} \subset(0, \infty)$ are two sequences converging to the same limit $L \geq 0$ and verifying that $L<a_{n}$ and $\varrho_{\phi}^{\prime}\left(a_{n}, b_{n}\right)>0$ for all $n \in \mathbb{N}$. In order to prove that $L=0$, assume that $L>0$. Therefore,

$$
0<\varrho_{\phi}^{\prime}\left(a_{n}, b_{n}\right)=\phi\left(b_{n}\right) b_{n}-a_{n} \quad \text { for all } n \in \mathbb{N} \text {. }
$$

As a consequence,

$$
L<a_{n}<\phi\left(b_{n}\right) b_{n} \leq b_{n} \quad \text { for all } n \in \mathbb{N} \text {. }
$$

Letting $n \rightarrow \infty$ in the last inequalities, we deduce that $\lim _{n \rightarrow \infty} \phi\left(b_{n}\right)=1$. As $\phi$ is a Geraghty function, it follows that $L=\lim _{n \rightarrow \infty} b_{n}=0$, which contradicts $L>0$. Then necessarily $L=0$.

$\left(\varrho_{3}\right)$ Let $\left\{a_{n}\right\},\left\{b_{n}\right\} \subset(0, \infty) \cap A$ be two sequences such that $\left\{b_{n}\right\} \rightarrow 0$ and $\varrho_{\phi}^{\prime}\left(a_{n}, b_{n}\right)>0$ for all $n \in \mathbb{N}$. Therefore $0<\varrho_{\phi}^{\prime}\left(a_{n}, b_{n}\right)=\phi\left(b_{n}\right) b_{n}-a_{n} \leq b_{n}-a_{n}$, so $0<a_{n}<b_{n}$ for all $n \in \mathbb{N}$, which implies that $\left\{a_{n}\right\} \rightarrow 0$. 


\subsection{R-Contractions}

In this section we introduce the notion of $R$-contraction and we show several examples of such kind of contractions.

Definition 24 Let $(X, d)$ be a metric space and let $T: X \rightarrow X$ be a mapping. We will say that $T$ is an $R$-contraction if there exists an $R$-function $\varrho: A \times A \rightarrow \mathbb{R}$ such that $\operatorname{ran}(d) \subseteq A$ and

$$
\varrho(d(T x, T y), d(x, y))>0 \quad \text { for all } x, y \in X \text { such that } x \neq y \text {. }
$$

In such a case, we will say that $T$ is an $R$-contraction with respect to $\varrho$. We denote the family of all $R$-contractions from $(X, d)$ into itself with respect to $\varrho$ by $R_{X, d, A, \varrho}$ or, when no confusion is possible, by $R_{\varrho}$.

The following result shows an extensive family of $R$-contractions.

Theorem 25 Every Meir-Keeler contraction is an R-contraction with respect to an $R$ function $\varrho$ which satisfies $\left(\varrho_{3}\right)$.

In the last result, the $R$-function $\varrho$ may be chosen satisfying the following properties.

- $\varrho(t, s)<s-t$ for all $t, s \in(0, \infty)$.

- $\varrho(t, s) \leq s-t$ for all $t, s \in[0, \infty)$.

Proof Let $(X, d)$ be a metric space and let $T: X \rightarrow X$ be a Meir-Keeler contraction. By Theorem 9, there exists an $L$-function $\phi$ such that

$$
d(T x, T y)<\phi(d(x, y)) \quad \text { for all } x, y \in X \text { verifying } d(x, y)>0 .
$$

Theorem 21 guarantees that $\varrho_{\phi}:[0, \infty) \times[0, \infty) \rightarrow \mathbb{R}$, defined by

$$
\varrho_{\phi}(t, s)=\phi(s)-t \quad \text { for all } t, s \in[0, \infty)
$$

is an $R$-function on $[0, \infty)$ which satisfies $\left(\varrho_{3}\right)$. Moreover, for all $x, y \in X$ such that $x \neq y$, we have that

$$
\varrho_{\phi}(d(T x, T y), d(x, y))=\phi(d(x, y))-d(T x, T y)>0,
$$

which means that $T$ is an $R$-contraction.

The previous statement implies that every fixed point theorem that can be proved for $R$-contractions (such as Theorem 27) also holds for Meir-Keeler contractions. However, the converse is false as we shall see in the next section.

Corollary 26 Every Geraghty contraction is an R-contraction with respect to an R-function $\varrho$ which satisfies $\left(\varrho_{3}\right)$. 
Proof Let $(X, d)$ be a metric space and let $T: X \rightarrow X$ be a Geraghty contraction. Then there exists a Geraghty function $\phi:[0, \infty) \rightarrow[0,1)$ such that

$$
d(T x, T y) \leq \phi(d(x, y)) d(x, y) \quad \text { for all } x, y \in X .
$$

Let $\varphi:[0, \infty) \rightarrow[0,1)$ be the function defined by $\varphi(t)=(1+\phi(t)) / 2$ for all $t \in[0, \infty)$. Then $\varphi$ is also a Geraghty function and it verifies $\phi(t)<\varphi(t)<1$ for all $t \in[0, \infty)$. Therefore, if $x \neq y$, then

$$
d(T x, T y) \leq \phi(d(x, y)) d(x, y)<\varphi(d(x, y)) d(x, y) .
$$

Lemma 23 guarantees that $\varrho_{\varphi}^{\prime}:[0, \infty) \times[0, \infty) \rightarrow \mathbb{R}$, defined by

$$
\varrho_{\varphi}^{\prime}(t, s)=\varphi(s) s-t \quad \text { for all } t, s \in[0, \infty)
$$

is an $R$-function on $[0, \infty)$ which satisfies $\left(\varrho_{3}\right)$. Moreover, for all $x, y \in X$ such that $x \neq y$, we have that $\phi(d(x, y))<1$ and

$$
\varrho_{\varphi}^{\prime}(d(T x, T y), d(x, y))=\varphi(d(x, y)) d(x, y)-d(T x, T y)>0,
$$

which means that $T$ is an $R$-contraction.

\section{Some fixed point theorems under $R$-contractivity conditions}

This section is dedicated to obtaining fixed point theorems under $R$-contractivity conditions. Later, we will show that some well-known results can be deduced as simple consequences of our main result, which is the following one.

Theorem 27 Let $(X, d)$ be a complete metric space and let $T: X \rightarrow X$ be an $R$-contraction with respect to $\varrho \in R_{A}$. Assume that, at least, one of the following conditions holds:

(a) $T$ is continuous.

(b) The function $\varrho$ satisfies condition $\left(\varrho_{3}\right)$.

(c) $\varrho(t, s) \leq s-t$ for all $t, s \in A \cap(0, \infty)$.

Then $T$ is a Picard operator. In particular, it has a unique fixed point.

Proof Let $x_{0} \in X$ be an arbitrary point and let $\left\{x_{n}\right\}_{n \geq 0}$ be the Picard sequence of $T$ based on $x_{0}$, that is, $x_{n+1}=T x_{n}$ for all $n \in \mathbb{N}$. If there exists some $n_{0} \in \mathbb{N}$ such that $x_{n_{0}+1}=x_{n_{0}}$, then $x_{n_{0}}$ is a fixed point of $T$. In the contrary case, assume that $x_{n} \neq x_{n+1}$ for all $n \in \mathbb{N}$. Let $\left\{a_{n}\right\} \subset(0, \infty)$ be the sequence defined by $a_{n}=d\left(x_{n}, x_{n+1}\right)>0$ for all $n \in \mathbb{N}$. Taking into account that $T$ is an $R$-contraction with respect to $\varrho$, then

$$
\varrho\left(a_{n+1}, a_{n}\right)=\varrho\left(d\left(x_{n+1}, x_{n+2}\right), d\left(x_{n}, x_{n+1}\right)\right)=\varrho\left(d\left(T x_{n}, T x_{n+1}\right), d\left(x_{n}, x_{n+1}\right)\right)>0 .
$$

Applying $\left(\varrho_{1}\right)$ we deduce that $\left\{d\left(x_{n}, x_{n+1}\right)\right\}=\left\{a_{n}\right\} \rightarrow 0$, that is, $\left\{x_{n}\right\}$ is an asymptotically regular sequence.

Next we show that $\left\{x_{n}\right\}$ is a Cauchy sequence reasoning by contradiction. If $\left\{x_{n}\right\}$ is not a Cauchy sequence, then there exist $\varepsilon_{0}>0$ and two subsequences $\left\{x_{n(k)}\right\}$ and $\left\{x_{m(k)}\right\}$ of $\left\{x_{n}\right\}$ 
such that

$$
\begin{aligned}
& k \leq n(k)<m(k), \quad d\left(x_{n(k)}, x_{m(k)-1}\right) \leq \varepsilon_{0}<d\left(x_{n(k)}, x_{m(k)}\right) \quad \text { for all } k \in \mathbb{N}, \\
& \lim _{k \rightarrow \infty} d\left(x_{n(k)}, x_{m(k)}\right)=\lim _{k \rightarrow \infty} d\left(x_{n(k)-1}, x_{m(k)-1}\right)=\varepsilon_{0} .
\end{aligned}
$$

Let $L=\varepsilon_{0}>0,\left\{a_{k}=d\left(x_{n(k)}, x_{m(k)}\right)\right\} \rightarrow L$ and $\left\{b_{k}=d\left(x_{n(k)-1}, x_{m(k)-1}\right)\right\} \rightarrow L$. Since $L=\varepsilon_{0}<$ $d\left(x_{n(k)}, x_{m(k)}\right)=a_{k}$ and

$$
\begin{aligned}
\varrho\left(a_{k}, b_{k}\right) & =\varrho\left(d\left(x_{n(k)}, x_{m(k)}\right), d\left(x_{n(k)-1}, x_{m(k)-1}\right)\right) \\
& =\varrho\left(d\left(T x_{n(k)-1}, T x_{m(k)-1}\right), d\left(x_{n(k)-1}, x_{m(k)-1}\right)\right)>0
\end{aligned}
$$

for all $k \in \mathbb{N}$, condition $\left(\varrho_{2}\right)$ guarantees that $\varepsilon_{0}=L=0$, which is a contradiction. As a consequence, $\left\{x_{n}\right\}$ is a Cauchy sequence. Since $(X, d)$ is complete, there exists $z \in X$ such that $\left\{x_{n}\right\} \rightarrow z$. Let us show that $z$ is a fixed point of $T$ distinguishing two cases.

Case 1 . Assume that $T$ is continuous. In this case, $\left\{x_{n+1}=T x_{n}\right\} \rightarrow T z$, so $T z=z$.

Case 2. Assume that the function $\varrho$ satisfies condition $\left(\varrho_{3}\right)$. In this case, let $a_{n}=d\left(x_{n+1}, T z\right)$ and $b_{n}=d\left(x_{n}, z\right)$ for all $n \in \mathbb{N}$. Then $\left\{b_{n}\right\} \rightarrow 0$. Moreover,

$$
\varrho\left(a_{n}, b_{n}\right)=\varrho\left(d\left(x_{n+1}, T z\right), d\left(x_{n}, z\right)\right)=\varrho\left(d\left(T x_{n}, T z\right), d\left(x_{n}, z\right)\right)>0
$$

for all $n \in \mathbb{N}$. Furthermore, it is clear that

$$
b_{n}=0 \quad \Rightarrow \quad a_{n}=0
$$

because

$$
b_{n}=0 \quad \Leftrightarrow \quad x_{n}=z \quad \Rightarrow \quad x_{n+1}=T x_{n}=T z \quad \Leftrightarrow \quad a_{n}=0 .
$$

Let us consider the set

$$
\Omega=\left\{n \in \mathbb{N}: a_{n}=0\right\}=\left\{n \in \mathbb{N}: d\left(x_{n+1}, T z\right)=0\right\} .
$$

Subcase 2.1. Assume that $\Omega$ is finite. In this case, there exists $n_{0} \in \mathbb{N}$ such that $d\left(x_{n+1}, T z\right)=a_{n}>0$ for all $n \geq n_{0}$. By (7), $d\left(x_{n}, z\right)=b_{n}>0$ for all $n \geq n_{0}$. Taking into account (6), condition $\left(\varrho_{3}\right)$, applied to $\left\{a_{n}\right\}_{n \geq n_{0}}$ and $\left\{b_{n}\right\}_{n \geq n_{0}}$, implies that $\left\{d\left(x_{n+1}, T z\right)=a_{n}\right\} \rightarrow 0$, which means that $\left\{x_{n+1}\right\} \rightarrow T z$. By the uniqueness of the limit, $T z=z$.

Subcase 2.2. Assume that $\Omega$ is not finite. In this case, there exists a subsequence $\left\{x_{n(k)}\right\}$ of $\left\{x_{n}\right\}$ such that

$$
d\left(x_{n(k)+1}, T z\right)=0 \quad \text { for all } k \in \mathbb{N} \text {. }
$$

Hence $x_{n(k)+1}=T z$ for all $k \in \mathbb{N}$. Since $\left\{x_{n}\right\} \rightarrow z$, then $T z=z$.

Case 3. Assume that $\varrho(t, s) \leq s-t$ for all $t, s \in A \cap(0, \infty)$. Proposition 14 implies that Case 2 is applicable.

In any case, $z$ is a fixed point of $T$. Then $T$ is a weakly Picard operator. 
Finally, let us show that $z$ is the unique fixed point of $T$. Let $x, y \in X$ be two arbitrary fixed points of $T$. We will deduce that $x=y$ reasoning by contradiction. Assume that $x \neq y$ and let us define $a_{n}=d(x, y)>0$ for all $n \in \mathbb{N}$. Therefore, for all $n \in \mathbb{N}$,

$$
\varrho\left(a_{n+1}, a_{n}\right)=\varrho(d(x, y), d(x, y))=\varrho(d(T x, T y), d(x, y))>0 .
$$

Applying $\left(\varrho_{1}\right),\left\{a_{n}\right\} \rightarrow 0$, which contradicts the fact that $d(x, y) \neq 0$. As a consequence, $x=y$ and $T$ has a unique fixed point.

Corollary 28 Every continuous $R$-contraction from a complete metric space into itself has a unique fixed point.

Corollary 29 Every $\mathcal{Z}$-contraction from a complete metric space into itself has a unique fixed point.

Corollary 30 Every $\widehat{\operatorname{Man}(\mathrm{R})}$-contraction from a complete metric space into itself has a unique fixed point.

Every manageable function leads to a simple corollary considering the corresponding $R$-function. For instance,

$$
\varrho(t, s)=\varphi(s)-s, \quad \varrho(t, s)=\phi(s)-\psi(t), \quad \text { etc. }
$$

Corollary 31 Let $(X, d)$ be a complete metric space and let $T: X \rightarrow X$ be a self-mapping. Assume that there exist two functions $\psi, \varphi:[0, \infty) \rightarrow[0, \infty)$ such that

$$
\psi(d(T x, T y)) \leq \psi(d(x, y))-\varphi(d(x, y)) \quad \text { for all } x, y \in X
$$

If $\psi$ is nondecreasing and continuous from the right, $\varphi$ is lower semi-continuous and $\varphi^{-1}(\{0\})=\{0\}$, then $T$ has a unique fixed point.

Proof If follows from Theorem 27 taking into account Theorem 22.

Corollary 32 Every Geraghty contraction from a complete metric space into itself has a unique fixed point.

Proof If follows from Theorem 27 taking into account Corollary 26.

Corollary 33 Every Meir-Keeler contraction from a complete metric space into itself has a unique fixed point.

Proof If follows from Theorem 27 taking into account Theorem 25.

Next, we show an example of an $R$-contraction which is not a Meir-Keeler contraction. Let $\mathbb{N}^{*}=\mathbb{N} \backslash\{0\}=\{1,2,3, \ldots\}$ and let $\left\{x_{n}\right\}$ and $\left\{y_{n}\right\}$ be the sequences

$$
x_{n}=10 n \quad \text { and } \quad y_{n}=10 n+1+\frac{1}{n} \quad \text { for all } n \in \mathbb{N}^{*} .
$$


Let $X$ be the set

$$
X=\{0,1\} \cup\left\{x_{n}\right\}_{n=1}^{\infty} \cup\left\{y_{n}\right\}_{n=1}^{\infty} \subset[0, \infty)
$$

endowed with the Euclidean metric $d(x, y)=|x-y|$ for all $x, y \in X$. As $X$ is discrete and

$$
d(x, y) \geq 1 \text { for all } x, y \in X \text { such that } x \neq y \text {, }
$$

then $(X, d)$ is complete. Let $T: X \rightarrow X$ be defined by

$$
T x= \begin{cases}0, & \text { if } x \in\{0,1\} \cup\{10 n\}_{n=1}^{\infty}, \\ 1, & \text { if } x \in\left\{10 n+1+\frac{1}{n}\right\}_{n=1}^{\infty} .\end{cases}
$$

Notice that $T$ satisfies the following properties:

- $T(X)=\{0,1\}$

- If $x, y \in X$ verify $d(T x, T y)>0$, then $d(T x, T y)=1$.

We claim that $T$ is an $R$-contraction but it is not a Meir-Keeler contraction. The second fact follows from Proposition 11 taking into account that

$$
d\left(T x_{n}, T y_{n}\right)=1<1+\frac{1}{n}=d\left(x_{n}, y_{n}\right) \quad \text { for all } n \in \mathbb{N}^{*} .
$$

Then $T$ is not a Meir-Keeler contraction. Next, we are going to show that $T$ is an $R$ contraction. To prove it, let $A=\operatorname{ran}(d) \subset[0, \infty)$ be the range of the metric $d$. As $X$ is numerable, then $A=d(X \times X)$ is also a numerable set. In fact, if $x, y \in X$ are such that $x \leq y$, then one, and only one, of the following cases holds:

$$
\begin{cases}\left(\mathrm{c}_{1}\right) & d(x, y)=0 \text { if, and only if, } x=y \\ \left(\mathrm{c}_{2}\right) & d(x, y)=1 \text { if, and only if, } x=0 \text { and } y=1 \\ \left(\mathrm{c}_{3}\right) & d(x, y)=1+\frac{1}{n} \text { if, and only if, } x=x_{n} \text { and } y=y_{n} \text { for some } n \in \mathbb{N}^{*} \\ \left(\mathrm{c}_{4}\right) & d(x, y) \geq 5 \text { in any other case. }\end{cases}
$$

As a consequence, the range of $d$ can be expressed as

$$
A=\operatorname{ran}(d)=\{0,1\} \cup\left\{1+\frac{1}{n}\right\}_{n=1}^{\infty} \cup\left\{\lambda_{k}\right\}_{k=0}^{\infty},
$$

where $\lambda_{k} \geq 5$ for all $k \in \mathbb{N}$. In particular,

$$
s \in A=d(X \times X), \quad s>0 \quad \Rightarrow \quad s \geq 1 .
$$

Let $\varrho: A \times A \rightarrow \mathbb{R}$ be the function given by

$$
\varrho(t, s)= \begin{cases}\frac{s}{2}, & \text { if } t=0 \\ \frac{s}{2}-t, & \text { if } s \geq 5 \text { and } t>0 \\ s-t, & \text { if } t=1 \text { and } s=1+\frac{1}{n} \text { for some } n \in \mathbb{N}^{*} \\ 0, & \text { otherwise. }\end{cases}
$$


Let us show that $\varrho$ is an $R$-function verifying condition $\left(\varrho_{3}\right)$. To do that, recall Remark 13 because we are going to show that there are not sequences as in the hypotheses of conditions $\left(\varrho_{1}\right),\left(\varrho_{2}\right)$ and $\left(\varrho_{3}\right)$.

$\left(\varrho_{1}\right)$ We claim that it is impossible to have a sequence $\left\{a_{n}\right\} \subset(0, \infty) \cap A$ such that $\varrho\left(a_{n+1}, a_{n}\right)>0$ for all $n \in \mathbb{N}$. To prove it, assume that such sequence exists. As $a_{n}>0$ for all $n \in \mathbb{N}$, description (11) leads to three cases.

Case 1. There exists $n_{0} \in \mathbb{N}$ such that $a_{n_{0}}=1$. As $t=a_{n_{0}+1}>0$ and $s=a_{n_{0}}=1<5$, then the inequality $\varrho\left(a_{n_{0}+1}, 1\right)=\varrho\left(a_{n_{0}+1}, a_{n_{0}}\right)>0$ is impossible following (13).

Case 2. There exists $n_{0} \in \mathbb{N}$ such that $a_{n_{0}}=1+\frac{1}{m}$ for some $m \in \mathbb{N}^{*}$. As $t=a_{n_{0}+1}>0$ and $s=a_{n_{0}}=1+\frac{1}{m}<5$, then necessarily $a_{n_{0}+1}=1$, but this is impossible by Case 1 .

Case 3. $a_{n} \geq 3$ for all $n \in \mathbb{N}$. In this case, for all $n \in \mathbb{N}$,

$$
0<\varrho\left(a_{n+1}, a_{n}\right)=\frac{a_{n}}{2}-a_{n+1} \quad \Rightarrow \quad a_{n+1} \leq \frac{a_{n}}{2} .
$$

Hence $\left\{a_{n}\right\} \rightarrow 0$ but this is impossible because, by (12), $a_{n} \geq 1$ for all $n \in \mathbb{N}$.

In any case, it is impossible to have a sequence $\left\{a_{n}\right\} \subset(0, \infty) \cap A$ such that $\varrho\left(a_{n+1}, a_{n}\right)>0$ for all $n \in \mathbb{N}$, which means that $\left(\varrho_{1}\right)$ holds.

$\left(\varrho_{2}\right)$ We claim that it is impossible to have two sequences $\left\{a_{n}\right\},\left\{b_{n}\right\} \subset(0, \infty) \cap A$ converging to the same limit $L \geq 0$ and verifying that $L<a_{n}$ and $\varrho\left(a_{n}, b_{n}\right)>0$ for all $n \in \mathbb{N}$. To prove it, assume that such sequences exist. Since $a_{n}>0$, then $a_{n} \geq 1$ for all $n \in \mathbb{N}$ by (12). Hence $1 \leq L<a_{n}$ for all $n \in \mathbb{N}$. The only case in which $\varrho(t, s)>0$ and $t>1$ occurs when $s \geq 5$. Thus $\varrho\left(a_{n}, b_{n}\right)>0$ implies that $b_{n} \geq 5$ for all $n \in \mathbb{N}$. Moreover, for all $n \in \mathbb{N}$,

$$
0<\varrho\left(a_{n}, b_{n}\right)=\frac{b_{n}}{2}-a_{n} \Rightarrow 2 a_{n}<b_{n} .
$$

Letting $n \rightarrow \infty$, we deduce that $0 \leq 2 L \leq L$, so $L=0$, which contradicts the fact that $L \geq 1$. This contradiction ensures that $\left(\varrho_{2}\right)$ holds.

$\left(\varrho_{3}\right)$ We claim that it is impossible to have two sequences $\left\{a_{n}\right\},\left\{b_{n}\right\} \subset(0, \infty) \cap A$ such that $\left\{b_{n}\right\} \rightarrow 0$ and $\varrho\left(a_{n}, b_{n}\right)>0$ for all $n \in \mathbb{N}$. To prove it, assume that such sequences exist. Since $b_{n}>0$, then $b_{n} \geq 1$ for all $n \in \mathbb{N}$ by (12), which contradicts the fact that $\left\{b_{n}\right\} \rightarrow 0$. Hence $\left(\varrho_{3}\right)$ holds.

As a consequence, $\varrho$ is an $R$-function on $A=\operatorname{ran}(d)$. Finally, we claim that $T$ is an $R$-contraction with respect to $\varrho$. Let $x, y \in X$ be such that $x \neq y$, that is, $d(x, y)>0$. If $d(T x, T y)=0$, then

$$
\varrho(d(T x, T y), d(x, y))=\varrho(0, d(x, y))=\frac{d(x, y)}{2}>0 .
$$

Suppose that $d(T x, T y)>0$. In this case, by (9) and (10), we have that $\{T x, T y\}=\{0,1\}$ and $d(T x, T y)=1$. Suppose that $T x=0$ and $T y=1$. Hence $x \in\{0,1\} \cup\{10 n\}_{n=1}^{\infty}$ and $y \in\{10 m+$ $\left.1+\frac{1}{m}\right\}_{m=1}^{\infty}$. Therefore, only cases $\left(\mathrm{c}_{3}\right)$ and $\left(\mathrm{c}_{4}\right)$ of (11) are possible. In case $\left(\mathrm{c}_{3}\right), x=x_{n}$ and $y=y_{n}$ for some $n \in \mathbb{N}^{*}$. Then

$$
\varrho(d(T x, T y), d(x, y))=\varrho\left(1,1+\frac{1}{n}\right)=\left(1+\frac{1}{n}\right)-1=\frac{1}{n}>0 .
$$


In case $\left(\mathrm{c}_{4}\right), d(x, y) \geq 5$, so

$$
\varrho(d(T x, T y), d(x, y))=\frac{d(x, y)}{2}-d(T x, T y) \geq \frac{5}{2}-1>1>0 .
$$

As a result, in any case, $\varrho(d(T x, T y), d(x, y))>0$ for all $x, y \in X$ such that $x \neq y$, so $T$ is an $R$-contraction with respect to $\varrho$.

As a consequence, Theorem 27 guarantees that $T$ has a unique fixed point. However, other previous results about Meir-Keeler contractions are not applicable.

\section{Competing interests}

The authors declare that they have no competing interests.

\section{Authors' contributions}

All authors contributed equally to the writing of this paper. All authors read and approved the final manuscript.

\section{Author details}

'Department of Quantitative Methods for Economics and Business, Facultad de Ciencias Económicas y Empresariales, University of Granada, Campus de Cartuja, Granada, Spain. ${ }^{2}$ Operator Theory and Applications Research Group, Department of Mathematics, King Abdulaziz University, P.O. Box 80203, Jeddah, 21589, Saudi Arabia.

\section{Acknowledgements}

This article was funded by the Deanship of Scientific Research (DSR), King Abdulaziz University, Jeddah. The second author, therefore, acknowledges with thanks DSR for technical and financial support. The authors are grateful to three anonymous referees for their useful suggestions and comments. AF Roldán López de Hierro is grateful to the Department of Quantitative Methods for Economics and Business of the University of Granada. The same author has been partially supported by Junta de Andalucía by project FQM-268 of the Andalusian CICYE.

Received: 19 March 2015 Accepted: 3 June 2015 Published online: 01 July 2015

\section{References}

1. Meir, A, Keeler, E: A theorem on contraction mappings. J. Math. Anal. Appl. 28, 326-329 (1969)

2. Lim, T-C: On characterizations of Meir-Keeler contractive maps. Nonlinear Anal. 46, 113-120 (2001)

3. Harjani, J, López, B, Sadarangani, K: A fixed point theorem for Meir-Keeler contractions in ordered metric spaces. Fixed Point Theory Appl. 2011, Article ID 83 (2011)

4. Jachymski, J: Equivalent conditions and the Meir-Keeler type theorems. J. Math. Anal. Appl. 194, 293-303 (1995)

5. Kadelburg, Z, Radenović, S: Meir-Keeler-type conditions in abstract metric spaces. Appl. Math. Lett. 24, 1411-1414 (2011)

6. Samet, B: Coupled fixed point theorems for a generalized Meir-Keeler contraction in partially ordered metric spaces. Nonlinear Anal. 72, 4508-4517 (2010)

7. Suzuki, T: Fixed-point theorem for asymptotic contractions of Meir-Keeler type in complete metric spaces. Nonlinear Anal. 64, 971-978 (2006)

8. Ding, HS, Karapınar, E: Meir-Keeler type contractions in partially ordered G-metric spaces. Fixed Point Theory Appl. 2013, Article ID 35 (2013)

9. Chi, KP, Karapınar, E, Thanh, TD: A generalization of the Meir-Keeler type contraction. Arab J. Math. Sci. 18, 141-148 (2012)

10. Samet, B: Coupled fixed point theorems for a generalized Meir-Keeler contraction in partially ordered metric spaces. Nonlinear Anal. 72, 4508-4517 (2010)

11. Karapınar, E, Roldán, A, Martínez-Moreno, J, Roldán, C: Meir-Keeler type multidimensional fixed point theorems in partially ordered metric spaces. Abstr. Appl. Anal. 2013, Article ID 406026 (2013)

12. Khojasteh, F, Shukla, S, Radenović, S: A new approach to the study of fixed point theory for simulation functions. Filomat 29(6), 1189-1194 (2015)

13. Roldán-López-de-Hierro, AF, Karapınar, E, Roldán-López-de-Hierro, C, Martínez-Moreno, J: Coincidence point theorems on metric spaces via simulation functions. J. Comput. Appl. Math. 275, 345-355 (2015)

14. Du, W-S, Khojasteh, F: New results and generalizations for approximate fixed point property and their applications. Abstr. Appl. Anal. 2014, Article ID 581267 (2014)

15. Găvruța, L, Găvruța, P, Khojasteh, F: Two classes of Meir-Keeler contractions. arXiv:1405.5034v1 [math.FA] 20 May 2014

16. Rus, IA: Weakly Picard operators and applications. Semin. Fixed Point Theory Cluj-Napoca 2, 41-58 (2001)

17. Rus, IA: Picard operators and applications. Sci. Math. Jpn. 58, 191-219 (2003)

18. Geraghty, M: On contractive mappings. Proc. Am. Math. Soc. 40, 604-608 (1973)

19. Chu, SC, Diaz, JB: Remarks on a generalization of Banach's principle of contraction mappings. J. Math. Anal. Appl. 2 , 440-446 (1965) 